\title{
INDEX NUMBERS AND THE GENERAL ECONOMIC EQUILIBRIUM*
}

\author{
F. W. DRESCH
}

What may be called the problem of imputation, which is a central problem of economics, is that of determining how the end product of the industrial process, called consumption goods, comes to be distributed among the individuals or classes of individuals which make up the economic system. Corresponding to the classes of individuals are classes of goods, which may in particular be arranged as follows:

1. Capital or production goods (goods used for the production of other goods).

2. Primary factors, principally labor and the service of lands.

3. Consumption goods.

In what follows, by means of indices of prices and productions which have a theoretical foundation, the state of a system is described in terms of these classes. The particular problem most completely discussed is that of equilibrium under strict competition.

1. Virtual equations of the equilibrium situation. Let $q_{1}, q_{2}, \cdots, q_{n}$ be the rates of production per unit time of $n$ commodities, the same or different, by $n$ different producers, the commodities selling at prices $p_{1}, p_{2}, \cdots, p_{n}$. These goods we suppose to be arranged in the three classes $1,2,3$, defined above. We assume first

$$
q_{i}=q_{i}\left(q_{j}^{i}\right)_{j \cdot 12},
$$

denoting by $q_{j}{ }^{i}$ the portion of $q_{j}$ which is used per unit time in the production of $q_{i}$, and meaning by (1) that the amount $q_{i}$ of any commodity of classes 1 or 3 , which is produced per unit time, is a "technical" function of the amounts of capital goods and primary factors devoted to it; that is, $q_{i}$ is a function of all the $q_{j}^{i}$ of classes 1 and 2 for any $i$ of classes 1 or 3 . Also we assume that

$$
q_{i}=\sum_{j \cdot 13} q_{i}^{j},
$$

meaning that all of any $q_{i}$ in class 1 or class 2 is portioned among the producers of goods of classes 1 and 3 . It is, in fact, a requirement of the equilibrium that no commodity is being stored up at any time $t$ in the various compartments of the system. In particular, as stated

* Presented to the Society, April 3, 1937. 
in (4), below, it is assumed that all the net incomes or profits are spent on consumption goods. The amounts or rates considered need not, however, be constant in time, for the equilibria may be moving equilibria.

Profits or net incomes are given by the formulas

$$
\begin{aligned}
& \pi_{i}=p_{i} q_{i}-\sum_{j \cdot 12} p_{j} q_{j}^{i}, \\
& \pi_{i}=p_{i} q_{i},
\end{aligned}
$$

so that, at a time $t$,

$$
d \pi_{i}=q_{i} d p_{i}-\sum_{j \cdot 12} q_{j}^{i} d p_{j}+\sum_{j \cdot 12}\left(p_{i} \frac{\partial q_{i}}{\partial q_{j}{ }^{i}}-p_{j}\right) d q_{j}{ }^{i},
$$

in virtue of the relations (1).

Consider now the case of strict competition, in which the producer supposes that the prices are not under his control, and endeavors to maximize the $\pi_{i}$ without considering changes in the $p$ 's. In that case, from (3),

$$
p_{i} \frac{\partial q_{i}}{\partial q_{j}^{i}}-p_{j}=0
$$

and these equations may be said to represent the producer's point of view and guide his use of capital and primary factors in the process of production. By means of them, equations (3) are reduced to the following:

$$
d \pi_{i}=q_{i} d p_{i}-\sum_{j \cdot 12} q_{j}^{i} d p_{j}
$$

which coordinate displacements from equilibrium, at time $t$.

The sum of the profits $\pi_{i}$ for the classes 1,3 is the quantity $\Pi=\sum_{i \cdot 13} \pi_{i}$. By means of $\left(3^{\prime}\right)$ and (2), its differential takes the form

$$
d \Pi=\sum_{i \cdot 3} q_{i} d p_{i}-\sum_{i \cdot 2} q_{i} d p_{i} .
$$

On the other hand, since all of these profits are spent on consumption goods, we have

$$
\Pi=V_{3}-V_{2}
$$

with $V_{3}=\sum_{i \cdot 3} p_{i} q_{i}, V_{2}=\sum_{i \cdot 2} p_{i} q_{i}$, by means again of (2). Hence from $\left(3^{\prime \prime}\right)$ and (4) we have

$$
\sum_{i \cdot 3} p_{i} d q_{i}=\sum_{i \cdot 2} p_{i} d q_{i}
$$


In particular, if restraints are introduced in order to state an assumption that primary factors are fully employed, that is, that the $q_{i}$ of the class 2 are given functions of $t$, we deduce the equation

$$
\sum_{i \cdot 3} p_{i} d q_{i}=0
$$

as the principle of virtual displacements for our economic system. It may be noted that these restraints do not need to be introduced prior to the equations $p_{i} \partial q_{i} / \partial q_{j}{ }^{i}=p_{j}$ which represent the plans of the producers.

2. Class indices. Indices may now be introduced into the system according to the Divisia formulas*

$$
\frac{d Q_{r}}{Q_{r}}=\frac{\sum_{i \cdot r} p_{i} d q_{i}}{\sum_{i \cdot r} p_{i} q_{i}}, \quad \frac{d P_{r}}{P_{r}}=\frac{\sum_{i \cdot r} q_{2} d p_{i}}{\sum_{i \cdot r} q_{i} p_{i}}, \quad r=1,2,3,
$$

in order to compare one state of the system with another, along any hypothetical path of transformation of the system (not necessarily the actual transformation in time, as with Divisia). They may be integrated along any path, to yield a function of paths; for instance,

$$
Q_{2}(\lambda)=Q_{2}\left(\lambda_{0}\right) \exp \left[\int_{\lambda_{0}}^{\lambda}\left(\sum_{i \cdot 2} p_{i} d q_{i} / V_{2}\right)\right], \quad V_{2}=\sum_{i \cdot 2} p_{i} q_{i},
$$

in which $\lambda$ is a parameter of transformation.

With these definitions, equation (6) may be written in the form

$$
d Q_{3}=0 ;
$$

that is, under the hypothesis of strict competition, with primary factors fully used, the index of consumption goods is extremal with respect to virtual displacements. Roughly speaking, consumption commodity is a maximum. In order to justify completely this last statement, it is necessary of course to consider second order differentials and insert additional minor hypotheses on the derivatives of the technical functions $q_{i}=q_{i}\left(q_{j}{ }^{i}\right)$.

Partial indices may be introduced by the definitions

$$
\left(d Q_{3}\right)_{1}=\frac{Q_{3}}{V_{3}} \sum_{i \cdot 3} \sum_{j \cdot 1} p_{i} \frac{\partial q_{i}}{\partial q_{j}{ }^{i}} d q^{i},
$$

*F. Divisia, Economique Rationnelle, Paris, 1928, p. 268. These are the only indices whose differentials are linear in the differentials of prices and quantities, such that price indices do not change when quantities alone change, and vice versa, and such that the product of price and quantity indices is an index of money value. 
this being the change in $Q_{3}$ due to changes in the amounts of capital goods used, and

$$
d Q_{1}{ }^{3}=\frac{Q_{1}}{V_{1}} \sum_{i \cdot 1} \sum_{j \cdot 3} p_{i} d q_{i}{ }^{j},
$$

which is the part of $d Q_{1}$ which goes into the manufacture of consumption goods $Q_{3}$. The quantity defined by the relation

$$
\frac{\partial Q_{3}}{\partial Q_{1}{ }^{3}}=\frac{\left(d Q_{3}\right)_{1}}{d Q_{1}{ }^{3}}
$$

is thus a partial rate of change, that of consumption goods index with respect to capital goods contributions. This and the other analogous partial derivatives of $Q_{3}$ and $Q_{1}$ may be shown to satisfy the equations

$$
\begin{aligned}
\frac{\partial Q_{1}}{\partial Q_{1}{ }^{1}} & =1, & \frac{\partial Q_{1}}{\partial Q_{2}{ }^{1}} & =\frac{V_{2}}{Q_{2}} / \frac{V_{1}}{Q_{1}}, \\
\frac{\partial Q_{3}}{\partial Q_{1}{ }^{3}} & =\frac{V_{1}}{Q_{1}} / \frac{V_{3}}{Q_{3}}, & \frac{\partial Q_{3}}{\partial Q_{2}{ }^{3}} & =\frac{V_{2}}{Q_{2}} / \frac{V_{3}}{Q_{3}}
\end{aligned}
$$

at equilibrium. By elimination we have also

$$
\frac{\partial Q_{3}}{\partial Q_{1}{ }^{3}} \frac{\partial Q_{1}}{\partial Q_{2}{ }^{1}}=\frac{\partial Q_{3}}{\partial Q_{2}{ }^{3}} .
$$

Since, from the definition of indices,

$$
\frac{P_{3} Q_{3}}{P_{30} Q_{30}}=\frac{V_{3}}{V_{30}},
$$

where the subscript 0 refers to the "base" situation, we have, if we take the "base" indices as unity, $V_{3} / Q_{3}=V_{30} P_{3}$. In other words, the quantities $V_{3} / Q_{3}$ are not prices, and in fact cannot be, since prices have dimensions which vary from quantity to quantity; nevertheless the equations bear an interesting relation to those for a simplified system based on capital, labor, and consumption goods as if they were three commodities, namely,

$$
\begin{array}{llll}
\frac{\partial q_{1}}{\partial q_{2}{ }^{1}}=\frac{p_{2}}{p_{1}}, & \frac{\partial q_{3}}{\partial q_{1}^{3}}=\frac{p_{1}}{p_{3}}, & \frac{\partial q_{3}}{\partial q_{2}{ }^{3}}=\frac{p_{2}}{p_{3}} \\
\frac{\partial q_{1}}{\partial q_{1}^{1}}=1, & \frac{\partial q_{3}}{\partial q_{1}^{3}} \frac{\partial q_{1}}{\partial q_{2}^{1}}=\frac{\partial q_{3}}{\partial q_{2}{ }^{3}}, & d q_{3}=0
\end{array}
$$


and justify discussion of such systems. ${ }^{*}$ In particular, the problem of imputation in the simplified system may be treated by solving these equations.

It may be noted in passing that from some points of view it is desirable to replace the parts of indices like $d Q_{1}{ }^{3}$ by true partial indices, for example, with the definitions

$$
\frac{d Q_{1}{ }^{3}}{Q_{1}{ }^{3}}=\frac{1}{V_{1}{ }^{3}} \sum_{i \cdot 1} \sum_{j \cdot 3} p_{i} d q_{i}^{j}, \quad V_{1}^{3}=\sum_{i \cdot 1} \sum_{j \cdot 3} p_{i} q_{i}{ }^{j} .
$$

These in fact lead to equations analogous to (9).

3. Monopoly and limited competition. Given that the equilibrium of strict competition satisfies the equations of virtual displacement (5), or (6) in the case of given amounts of primary factors, one would expect an equilibrium in which exist limited forms of competition or monopoly to be expressed in a modified form of these virtual equations. In fact the latter forms of production are possible if the producer makes some sort of hypothesis about the relation of demand for his product to price, since he is not assuming that the price is outside the scope of his manipulation. In particular, the monopolist assumes that he knows the "elasticity of demand," $e_{i}$, in the relation

$$
p_{i} d q_{i}=-e_{i} q_{i} d p_{i}
$$

instead of assuming that $d p_{i}=0$ when he plans to maximize his profit. This assumption leads to the equations

$$
p_{i} \frac{\partial q_{i}}{\partial q_{j}}=r_{i} p_{j}, \quad r_{i}=\frac{e_{i}}{e_{i}-1}, \quad(i \cdot 13)(j \cdot 12),
$$

which govern the planning of the monopolist in regard to the relation between his own price and production, provided that he still considers the prices involved in his cost function as given. Thus the portion of $d \pi_{i}$ which does not involve $d p_{j},(j \cdot 12)$, is made to vanish, and

$$
p_{i} d q_{i}+q_{i} d p_{i}-\sum_{j \cdot 12} p_{j} d q_{j}^{i}=0
$$

which with (10) yields $\left(10^{\prime}\right)$. By summation, we have

* G. C. Evans, Maximum production studied in a simplified economic system, Econometrica, vol. 2 (1934), pp. 37-50.

O. Lange, The place of interest in the theory of production, Review of Economic Studies, vol. 3 (1936), pp. 159-192. The equations involving $p_{3}$ for the simplified system were first published by Lange, but were used independently in The University of California seminar on mathematical economics. 


$$
\sum_{i \cdot 13}\left(p_{i} d q_{i}+q_{i} d p_{i}\right)=\sum_{j \cdot 12} p_{i} d q_{i}
$$

If the assumption of the monopolist is correct, the equations (10) may be taken as additional restraints on the system, and (11) becomes then a principle of virtual displacements. It contains (5) formally, as is seen by putting the $r_{i}$ equal to unity. A similar modification occurs also in the equations (9). Thus the coefficient $R_{w}{ }^{u}$, as different from unity, in equations of the type

$$
\frac{\partial Q_{u}}{\partial Q_{w}^{u}}=R_{w}{ }_{w} \frac{V_{w}}{Q_{w}} / \frac{V_{u}}{Q_{u}}
$$

measures the departure from strict competition. The $u, w$ may refer to subclasses of the main classes $1,2,3$.

The relations so far treated have been obtained without the necessity of a full determination of the coordinates $q_{i}{ }^{j}, p_{i}$, as a count of the equations will show. To complete the picture of the equilibrium situation, demand relations in general may be given, say the statistically determined fraction $f_{i j}$ of $\pi_{j}$ which is spent on $q_{i},(i \cdot 3)$.

4. Equilibrium theory of interest. As an application of the theory consider the problem of the rate of interest in a situation of strict competition under equilibrium conditions. Although the interesting problem should be dynamic rather than static, we can think of the phenomena in the equilibrium case as due to the necessity of using money in production, money being owned by a special class, as if it were a primary factor like land and labor. The price of renting it, which is the rate of interest, will appear in the same category as the rent of land and the wages of labor in class 2 .

But it is also convenient to introduce the rate of interest, with the same ultimate result, in terms of restraints on the system, on postulating that the total cost of production is accepted by each producer as limited to a certain amount; that is,

$$
\sum_{j \cdot 12} p_{j} q_{j}^{i}=K_{i}
$$

In this case, we maximize, with respect to choices of the $q_{j}^{i}$, the expressions

$$
I_{i}=p_{i} q_{i}-\sum_{j \cdot 12} p_{j} q_{j}^{i}=p_{i} q_{i}-\sum_{j \cdot 12} p_{j} q_{j}^{i}+\lambda_{i}\left[K_{i}-\sum_{j \cdot 12} p_{j} q_{j}^{i}\right],
$$

the restraints entering through the Lagrangian multipliers $\lambda_{i}$. Thus we obtain the equations 


$$
p_{i} \frac{\partial q_{i}}{\partial q_{j}{ }^{i}}-\left(1+\lambda_{i}\right) p_{j}=0,
$$

with the hypothesis of strict competition. Here the $\lambda_{i}$ are not necessarily elements of cost, but the equilibrium conditions (12) are the same as if they were such elements, appearing as rates of interest. We should then define the profits by the formulas

$$
\pi_{i}=I_{i}-\lambda_{i} K_{i},
$$

and as a first approximation we might assume the $\lambda_{i}$ all equal, $\lambda_{i}=\lambda$.

The same formal result may be obtained by making extremal (at any time, $t=t_{0}$ ) the quantity

$$
I=\sum_{i \cdot 13} p_{i} q_{i}-\sum_{j \cdot 12} p_{j} q_{j}
$$

subject to the restraints

$$
\sum_{j \cdot 12} p_{j} q_{j}=K=K\left(t_{0}\right)
$$

with all the $d p_{i}$ set equal to zero; in fact, there appears in this way a single $\lambda$. Between $I$ and the value $\Pi=\sum_{i \cdot 13} \pi_{i}$ there holds the relation $I=\Pi+\lambda K$.

In terms of the indices of prices and quantities, the equation (9) becomes

$$
\frac{V_{u}}{Q_{u}} \frac{\partial Q_{u}}{\partial Q_{w}^{u}}=(1+\lambda) \frac{V_{w}}{Q_{w}},
$$

so that for the three fundamental categories, with the various $V_{u}, V_{w}$ eliminated, we have the equations

$$
\begin{aligned}
\frac{\partial Q_{1}}{\partial Q_{1}{ }^{1}} & =1+\lambda, \\
\lambda & =\frac{\frac{\partial Q_{3}}{\partial Q_{1}{ }^{3}} \frac{\partial Q_{1}}{\partial Q_{2}{ }^{1}}-\frac{\partial Q_{3}}{\partial Q_{2}{ }^{3}}}{\frac{\partial Q_{3}}{\partial Q_{2}{ }^{3}}} .
\end{aligned}
$$

The second of these equations states that $1+\lambda$ is equal to the ratio of the marginal productivity of indirect factors of production (that is, productive of consumption goods indirectly through the production of capital goods) to the marginal productivity of direct factors of production. The equation is essentially the formula for the rate of 
interest given by Wicksell.* It was obtained for the simplified system already mentioned, by Lange. $\dagger$ The remarks in this section are in fact a generalization or justification of this latter theory.

The index relations are particularly interesting in discussing changes from one system to another consequent on the introduction or change of interest rate $\lambda$. Thus, with the index of primary factors given, that is, $Q_{2}$ given, the introduction of a small interest rate $\lambda$ induces no modification of $Q_{3}$ as far as differentials of the first order. In fact,

$$
\delta Q_{3}=0
$$

and

$$
\delta^{2} Q_{3}=\lambda \frac{\partial Q_{3}}{\partial Q_{1}{ }^{3}} \delta Q_{1} .
$$

Equations such as these are important for economic theory.

University of CALifornia

\section{NOTE ON ALMOST-UNIVERSAL FORMS $\ddagger$}

\section{P. R. HALMOS}

Ramanujan§ and Dickson\| proved that there are 54 universal forms $a x^{2}+b y^{2}+c z^{2}+d t^{2}$ with positive integral coefficients $a, b, c, d$. It is the purpose of this note to investigate almost-universal forms, that is, to exhibit sets of positive integral coefficients $a, b, c, d$ such that $a x^{2}+b y^{2}+c z^{2}+d t^{2}$ represents every positive integer with exactly one exception.

Ramanujan§ showed that a necessary and sufficient condition that a form $a x^{2}+b y^{2}+c z^{2}+d t^{2}$ be universal is that it represent the first fifteen positive integers. Consequently the integer which an almostuniversal form fails to represent cannot be greater than 15 . Using Ramanujan's method of bounding the coefficients we can exhibit, merely by requiring that a form fail to represent exactly one of the

* Wicksell, Lectures on Political Economy, London, 1935 (translation), vol. 1, p. 156.

$\uparrow$ Lange, loc. cit.

$\ddagger$ Presented to the Society, December 28, 1937.

$\S$ Proceedings of the Cambridge Philosophical Society, vol. 19 (1917), pp. 11-21; Collected Papers, Cambridge, 1927, pp. 169-178.

|| This Bulletin, vol. 33 (1927), pp. 63-70. 